\title{
Supraciliary keyhole craniotomy for brain abscess debridement
}

\author{
Joseph C. Noggle, B.S., Daniel M. Sciubba, M.D., Clarke Nelson, B.S., \\ Giannina L. Garcés-AMbrossi, B.S., EDWARd AhN, M.D., AND GeORGe I. Jallo, M.D. \\ Department of Neurosurgery, Johns Hopkins School of Medicine, Baltimore, Maryland
}

\begin{abstract}
Object. Treatments for brain abscesses have typically involved invasive craniotomies followed by debridement. These methods often require large incisions with vast exposure and may be associated with high morbidity rates. For supraorbital lesions of the anterior and middle cranial fossa, minimally invasive craniotomies may limit exposure and decrease surgically related morbidity while allowing adequate debridement and decompression. The authors report their experience in treating frontal epidural abscesses in pediatric patients through minimally invasive supraciliary craniotomies over a 4-year period.

Methods. Three pediatric patients with frontal epidural abscesses underwent minimally invasive debridement procedures. Each procedure consisted of a supraciliary incision and a small craniotomy to expose the abscess. All patients underwent pre- and postoperative radiological evaluation including computed tomography and magnetic resonance imaging. Data were collected on preoperative characteristics, operative management, and postoperative outcomes.

Results. Two patients were male and 1 patient was female. The ages of the patients ranged from 6 to 10 years (mean 8 years). A frontal abscess was diagnosed in all patients, and all were treated surgically without perioperative complications. Microbes cultured postoperatively included methicillin-resistant Staphylococcus aureus in 2 patients and Staphylococcus viridans in 1 patient. The mean follow-up duration was 12.3 months. No neurological or vascular complications were noted during follow-up. All patients were treated with antibiotics postoperatively and experienced resolution of symptoms and excellent outcomes.

Conclusions. Frontal epidural abscesses can be adequately and safely debrided via a minimally invasive supraciliary craniotomy. This approach has a cosmetic benefit and may decrease approach-related morbidity.
\end{abstract} (DOI: 10.3171/FOC/2008/24/6/E11)

\author{
KeY WoRDS • brain abscess • debridement • eyebrow • minimally invasive \\ supraciliary keyhole craniotomy
}

I

NTRACRANIAL abscesses typically present with increased intracranial pressure, seizures, and focal neurological deficits and lead to significant morbidity., ${ }^{2,-9,13}$ These abscesses typically result from trauma, surgery, and hematogenous or local spread of infection. ${ }^{7}$ In the pediatric population, frontal abscesses are commonly the result of sinus infections.., 7

Although standard treatment of intracranial abscesses has included debridement in combination with intravenous antibiotics, such surgical drainage may be associated with substantial surgical morbidity., ${ }^{2,-9}$ Not only do craniotomies for abscess removal involve all of the risks typically associated with this procedure (such as stroke, brain damage, meningitis, hematomas, and others), ${ }^{7,8}$ but in situations in which cerebritis has not progressed to a well-formed abscess, corticotomy procedures may be associated with iatrogenic brain damage. In cases in which frontal sinusitis leads to intracranial abscesses of the anterior cranial fossa,

Abbreviation used in this paper: MRSA = methicillin-resistant Staphylococcus aureus. minimally invasive approaches may provide adequate decompression and debridement while minimizing approachrelated morbidity. ${ }^{13}$

In this paper, we describe the presentations and outcomes of 3 pediatric patients with intracranial abscesses who were treated using minimally invasive methods. Specifically, using a supraciliary incision with small frontal craniotomies, patients with intracranial abscesses can be treated with minimal surgically related morbidity.

\section{Clinical Materials and Methods}

A retrospective review of pediatric patients who underwent intracranial abscess debridement was conducted at a single institution over a 4-year period (January 2004 to January 2008). Patients treated using minimally invasive procedures were identified and evaluated. Data were collected on preoperative characteristics, operative management, and postoperative outcomes. All patients underwent preoperative and postoperative clinical and radiographic evaluation. Operative management consisted of a supracil- 


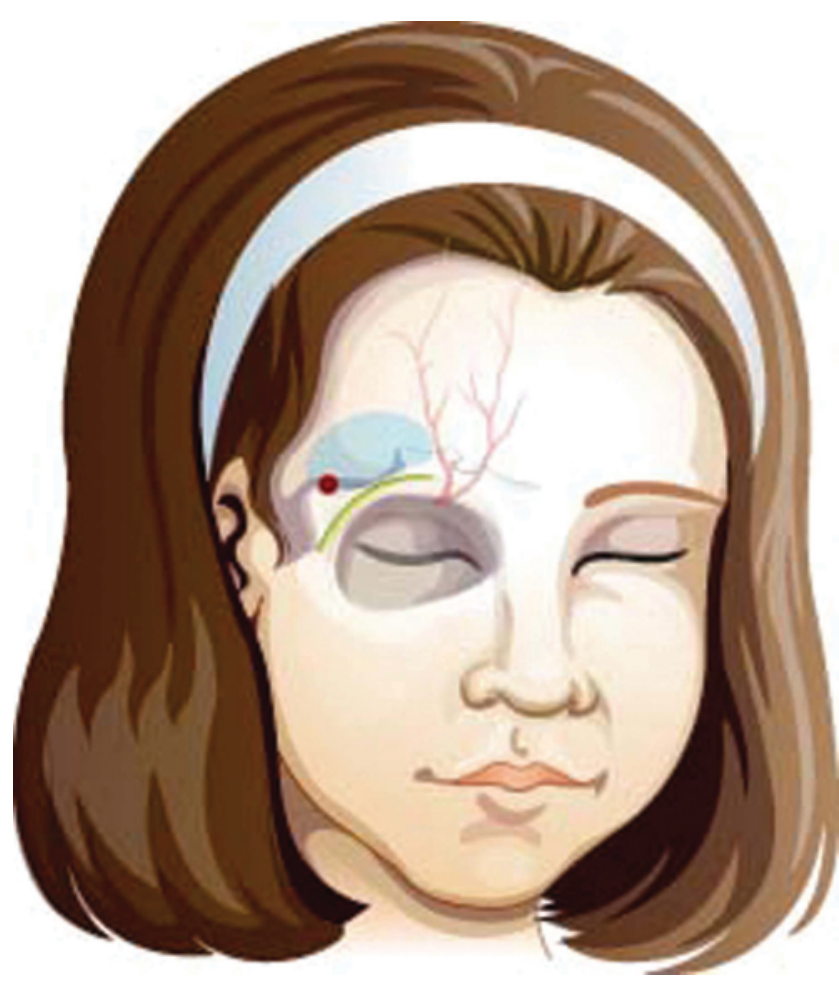

FIG. 1. Illustration of the orientation for a minimally invasive craniotomy (blue) through a supraciliary eyebrow incision (green). The red dot represents the bur hole. This approach exposes the anterior and middle cranial fossa.

iary incision and a minimally invasive frontolateral keyhole craniotomy, in which a small bur hole was drilled and widened (Fig. 1). After exposure and visualization, the lesions were debrided. In each procedure, a 1/8-inch drain was placed in the patient following abscess evacuation.

\section{Results}

Thirteen pediatric patients were treated for brain abscesses from January 2004 to January 2008. Six of these patients were treated nonoperatively using intravenous antibiotics, and 7 underwent surgical debridement. From the surgical group, 3 patients were treated using minimally invasive procedures.

Two boys and 1 girl with an average age of 8 years (range 6-10 years) underwent minimally invasive craniotomies for debridement. A right frontal epidural abscess was diagnosed in all patients, all of whom presented with headache, facial swelling, fever, and nausea. All patients were predisposed to abscess by a local infection, 1 from tooth infection and sinusitis, and 2 from sinusitis alone.

All patients were treated using surgical debridement through a minimally invasive craniotomy via the frontolateral supraciliary keyhole approach. Microbes cultured postoperatively included MRSA in 2 patients and Staphylococcus viridans in 1. Patients were followed for an average of 12.3 months (range 5-20 months). All patients were treated postoperatively with intravenous antibiotics, and all experienced resolution of symptoms and excellent outcomes.
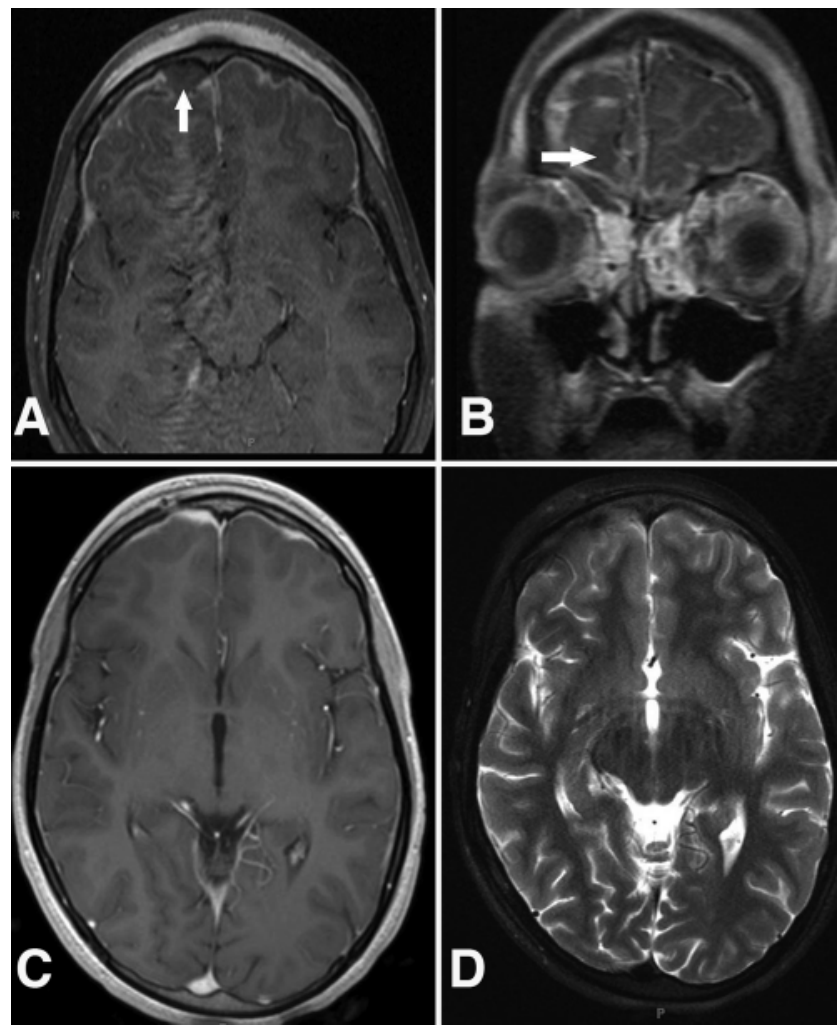

FIG. 2. Preoperative (A and B) and postoperative (C and D) MR images of a patient with a brain abscess. A and B: Axial (A) and coronal (B) T1-weighted images with gadolinium enhancement showing a right frontal abscess (arrow), right leptomeningeal enhancement, and bilateral enhancement of infected dura. C and D: Axial T1-weighted image with gadolinium enhancement $(C)$ and T2-weighted image (D) showing resolution of the right frontal abscess.

\section{Illustrative Case}

Presentation and Examination. This 10-year-old girl presented with facial swelling, headache, fever, and nausea 2 months after extraction of an infected tooth. She had no other history of prior illness. The patient was brought to the clinic in considerable pain, with erythema and moderate swelling around the left eye. She had dilated pupils and left-side superior rectus palsy.

Brain MR imaging with gadolinium enhancement revealed a $2.0 \times 1.2 \times 1.6-\mathrm{cm}$ empyema anterior to the right frontal pole and ethmoid sinusitis. The right frontal region displayed focal leptomeningeal enhancement, and extensive enhancement of the dura covered both cerebral convexities, suggesting infectious meningitis. Findings also included bilateral periorbital cellulitis, left postseptal cellulitis, and subperiosteal abscess along the superior medial orbital roof.

Operation and Postoperative Course. A decision was made by the neurosurgical team to aspirate the right epidural abscess via a supraciliary craniotomy. The left orbital cellulitis was treated with the assistance of the otolaryngology team, who dissected the medial and superior segments of the left orbit and drained the subperiosteal abscess. To access the ethmoid sinus for inspection and debridement, 
the otolaryngology team used an endoscopic approach through the maxillary sinus. Upon entrance into the maxillary sinus, a standard exploration was performed to orient the endoscope and to visualize the point of excision and the entrance point into the left ethmoid and frontal sinuses. Thickened mucous membranes were found in each location, and an infected fluid collection was removed from the ethmoid sinus (Fig. 2A and B).

After the right orbit was prepared and draped, a supraciliary incision was made extending $2 \mathrm{~cm}$ laterally from the medial edge of the eyebrow and deepened using Bovie cautery while preserving the supraorbital nerve. A bur hole was then drilled and widened, and a small craniotomy, roughly $2.5 \mathrm{~cm} \times 3.0 \mathrm{~cm}$ in size, was performed. Brownish fluid was immediately visible and aspirated under copious irrigation. Subperiosteal dissection of the subgaleal space was performed to further evacuate pus, and the area was washed with antibiotic irrigation. A 1/8-inch drain was placed in the patient before closure. Following surgery, abscess fluid was analyzed and found to host MRSA (Fig. 2C and D).

The postoperative course of the patient was uneventful. After a 2-month course of intravenous vancomycin and ceftriaxone, the patient returned to the clinic in excellent health with resolution of all symptoms. Upon examination, her incision had healed well, and she had good movement of her eyebrow with all cranial nerves intact.

\section{Discussion}

Intracranial abscesses lead to significant morbidity and are currently treated using antibiotics and surgery. Unfortunately, using only intravenous antibiotics may be associated with inadequate treatment and surgical debridement of the abscess may be associated with its own approachrelated morbidity. ${ }^{4,6,14,17,22}$ In this report we describe the presentation and outcomes of 3 pediatric patients who were treated for abscess drainage using minimally invasive procedures to outline a potential definitive treatment that can be associated with a lower surgical risk than a traditional approach.

Eyebrow craniotomies have been described for multiple types of intracranial pathologies (such as aneurysms, tumors, fractures, and infections).$^{18}$ Axel Perneczky has performed extensive work in this field, and has used the supraorbital subfrontal approach through an eyebrow craniotomy since 1985. Perneczky and his colleague Robert Reisch have described their experience with supraorbital procedures over a 10-year period. ${ }^{17}$ In their experiences using this approach, patients were treated for cerebral aneurysms, intracranial tumors or cysts, and other less frequently occurring diseases. The authors reported that supraorbital craniotomy permits adequate exposure of various intracranial entities, including those that are deep-seated, extended, or positioned bilaterally. Furthermore, compared with a traditional craniotomy, brain exposure and retraction are limited in their approach, thus reducing approach-related morbidity.

Recently, Jallo and colleagues ${ }^{10,11}$ described the frontolateral keyhole craniotomy procedure via a supraciliary skin incision in pediatric patients. This approach involves a small $2.5 \times 3.0-\mathrm{cm}$ bone opening directly above the eyebrow, which, as in the patient population of Perneckzky, decreases exposure and may reduce surgical morbidity. In his experience with 27 patients who ranged in age from 1 to 16 years, Jallo found the approach provided a simple and safe treatment for suprasellar and anterior cranial fossa lesions. Furthermore, he reported a postsurgical cosmetic benefit resulting from the location of the incision.

Many previously described subfrontal craniotomies require vast soft tissue dissection, bone exposure, and brain retraction. These approaches may introduce approach-related complications.4,6,14,17,22 After applying the technique described by Jallo, Perneckzky, and others to brain abscess treatment in children, we found that our outcomes are consistent with the decrease in surgery-related morbidity previously reported. Children who develop purulent sinusitis are at risk for developing intracranial abscesses, most commonly epidural in location, as a result of local spread of infection. ${ }^{5}$ All 3 of our patients had sinusitis and epidural purulent collections. Although all patients were concomitantly treated using intravenous antibiotics and with surgical sinus debridement performed by the otolaryngology service, definitive drainage of the intracranial abscess was not possible without an eventual craniotomy procedure. All patients had favorable intra- and perioperative courses, with complete resolution of the abscess and symptoms, no neurological complications, no further complications from infection, and no recurrence of the abscess.

Further advancements in the current treatment of brain abscesses may evolve from improvements in imaging, endoscopic navigation, and instrumentation. Preoperative and intraoperative imaging play a crucial role in determining abscess location and boundaries. ${ }^{17,18}$ Endoscopic naviga$\operatorname{tion}^{15,17}$ and the development of novel keyhole-specific in-

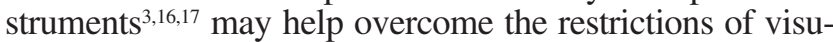
alization and instrument maneuverability in noninvasive craniotomy procedures. Furthermore, percutaneous techniques may provide an additional treatment modality and help to improve outcomes in abscess evacuation procedures. Open MR imaging systems with compatible instruments and fluoroscopy allow immediate imaging with high

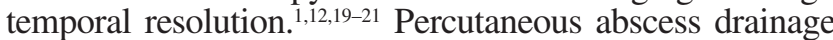
based on this technology has been demonstrated to be safe and reliable and has already been used to treat abscesses of the frontal lobe, parietal lobe, occipital lobe, temporal lobe, and basal ganglia. ${ }^{21}$

\section{Conclusions}

Frontal epidural abscesses can be adequately and safely debrided via a minimally invasive frontolateral keyhole craniotomy through a supraciliary skin incision. This approach has a cosmetic benefit, limits surgical exposure, and may decrease morbidity and improve outcomes for patients with frontal epidural abscesses.

\section{References}

1. Bernays RL, Kollias SS, Yonekawa Y: Dynamic changes during evacuation of a left temporal abscess in open MRI: technical case report. Neuroradiology 44:438-442, 2002

2. Fischer EG, McLennan JE, Suzuki Y: Cerebral abscess in children. Am J Dis Child 135:746-749, 1981

3. Fries G, Reisch R: Biportal neuroendoscopic microsurgical approaches to the subarachnoid cisterns. A cadaver study. Minim Invasive Neurosurg 39:99-104, 1996 
4. Fujitsu K, Kuwabara T: Zygomatic approach for lesions in the interpeduncular cistern. J Neurosurg 62:340-343, 1985

5. Glickstein JS, Chandra RK, Thompson JW: Intracranial complications of pediatric sinusitis. Otolaryngol Head Neck Surg 134: 733-736, 2006

6. Gonzalez LF, Crawford NR, Horgan MA, Deshmukh P, Zabramski JM, Spetzler RF: Working area and angle of attack in three cranial base approaches: pterional, orbitozygomatic, and maxillary extension of the orbitozygomatic approach. Neurosurgery 50:550-557, 2002

7. Goodkin HP, Harper MB, Pomeroy SL: Intracerebral abscess in children: historical trends at Children's Hospital Boston. Pediatrics 113:1765-1770, 2004

8. Hegde AS, Venkataramana NK, Das BS: Brain abscess in children. Childs Nerv Syst 2:90-92, 1986

9. Idriss ZH, Gutman LT, Kronfol NM: Brain abscesses in infants and children: current status of clinical findings, management and prognosis. Clin Pediatr (Phila) 17:738-740, 745-746, 1978

10. Jallo GI, Bognár L: Eyebrow surgery: the supraciliary craniotomy: technical note. Neurosurgery 59 (1 Suppl): ONSE157ONSE158, 2006

11. Jallo GI, Suk I, Bognar L: A superciliary approach for anterior cranial fossa lesions in children. Technical note. J Neurosurg 103 (1 Suppl):88-93, 2005

12. Kollias SS, Bernays RL: Interactive magnetic resonance imagingguided management of intracranial cystic lesions by using an open magnetic resonance imaging system. J Neurosurg 95:15-23, 2001

13. Longatti P, Perin A, Ettorre F, Fiorindi A, Baratto V: Endoscopic treatment of brain abscesses. Childs Nerv Syst 22:1447-1450, 2006

14. Maroon JC, Kennerdell JS: Surgical approaches to the orbit. Indications and techniques. J Neurosurg 60:1226-1235, 1984
15. Perneczky A, Fries G: Endoscope-assisted brain surgery: part 1evolution, basic concept, and current technique. Neurosurgery 42:219-225, 1998

16. Perneczky A, Fries G: Use of a new aneurysm clip with an inverted-spring mechanism to facilitate visual control during clip application. Technical note. J Neurosurg 82:898-899, 1995

17. Reisch R, Perneczky A: Ten-year experience with the supraorbital subfrontal approach through an eyebrow skin incision. Neurosurgery 57:242-255, 2005

18. Reisch R, Perneczky A, Filippi R: Surgical technique of the supraorbital key-hole craniotomy. Surg Neurol 59:223-227, 2003

19. Sakarya ME, Unal O, Ozbay B, Uzun K, Kati I, Ozen S, et al: MR fluoroscopy-guided transthoracic fine-needle aspiration biopsy: feasibility. Radiology 228:589-592, 2003

20. Tronnier V, Staubert A, Wirtz R, Knauth M, Bonsanto M, Kunze S: MRI-guided brain biopsies using a 0.2 Tesla open magnet. Minim Invasive Neurosurg 42:118-122, 1999

21. Unal O, Sakarya ME, Kiymaz N, Etlik O, Kayan M, Kati I, et al: Brain abscess drainage by use of MR fluoroscopic guidance. AJNR Am J Neuroradiol 26:839-842, 2005

22. Zabramski JM, Kiris T, Sankhla SK, Cabiol J, Spetzler RF: Orbitozygomatic craniotomy. Technical note. J Neurosurg 89:336-341, 1998

Manuscript submitted January 15, 2008.

Accepted March 14, 2008.

Address correspondence to: Daniel M. Sciubba, M.D., Department of Neurosurgery, Johns Hopkins University, 600 North Wolfe Street, Meyer 8-161, Baltimore, Maryland 21287. email: dsciubb1@jhmi.edu. 